\title{
Pericardial Effusion of Obscure Origin
}

\author{
Ramya Muralimohan · Adam Delu • \\ Thomas Ma
}

Received: 2 November 2014/ Accepted: 5 November 2014/Published online: 12 November 2014

(C) The Author(s) 2014. This article is published with open access at Springerlink.com

\section{Case Presentation}

A 41-year-old man was initially evaluated in the emergency department for a 2-month history of shortness of breath, abdominal distension, scrotal swelling, and left inguinal pain. Past history included long-term use of intravenous drugs, extensive and current alcohol use with evidence of cirrhosis based on laboratory findings, and imaging and seropositivity for hepatitis $\mathrm{C}$ antibody without measurable viremia. He also had a history of sustaining an upper abdominal stab wound requiring exploratory laparotomy in the early 1990s. He denied fevers, rigors, night sweats, weight loss, or skin rashes.

Initial evaluation revealed an ill appearing man with tachycardia (heart rate 120 beats/min) with mild hypotension (blood pressure 85-105/50-60 mmHg), and tachypnea (respiratory rate $20-25 / \mathrm{min}$ ) requiring $2 \mathrm{~L} / \mathrm{min}$ supplemental oxygen by nasal cannula to maintain a blood oxygen saturation $>95 \%$. He had distant heart sounds with tachycardia and regular rhythm without murmurs or gallops. He had jugular venous distension with a positive Kussmaul's sign and pulsus paradoxus. There was significant abdominal distention present with a fluid wave. He had stigmata of chronic liver disease with spider angiomata noted and collateral venous distension in the abdominal

\section{R. Muralimohan $(\varangle) \cdot$ T. Ma}

Division of Gastroenterology and Hepatology, Department of Internal Medicine, MSC10-5550, 1 University of New Mexico, University of New Mexico Health Sciences Center,

Albuquerque, NM 87131, USA

e-mail: rmuralimohan@salud.unm.edu

A. Delu

Department of Interventional Radiology, University of New Mexico Health Sciences Center, Albuquerque, NM, USA wall. Furthermore, he had scrotal swelling and bilateral lower extremity edema. He was admitted to the hospital's Medicine Service.

On admission, a chest radiograph revealed enlargement of the cardiac silhouette (Fig. 1). A bedside echocardiogram illustrated a large pericardial effusion with evidence of hemodynamic compromise, including right atrial and right ventricular diastolic collapse, a $>30 \%$ respiratory variability in the tricuspid and mitral valve flow rates, and a swinging cardiac motion consistent with the presence of cardiac tamponade demanding urgent intervention.

Admission laboratory values revealed a normal white blood cell count, a mild macrocytic anemia (mean corpuscular volume $95 \mathrm{fL}$ ) with a hemoglobin of $12.1 \mathrm{~g} / \mathrm{dL}$, a hematocrit of $36 \%$, and thrombocytopenia with a platelet count of $84,000 / \mu \mathrm{L}$. Tests of liver function revealed serum concentrations of albumin of $2.2 \mathrm{~g} / \mathrm{dL}(N>3.5 \mathrm{~g} / \mathrm{dL})$, total bilirubin of $3.1 \mathrm{mg} / \mathrm{dL}$, with direct bilirubin of $1.9 \mathrm{mg} / \mathrm{dL}$, an aspartate aminotransferase of $83 \mathrm{U} / \mathrm{mL}$, an alanine aminotransferase of $37 \mathrm{U} / \mathrm{mL}$, an alkaline phosphatase of $106 \mathrm{U} / \mathrm{mL}$ (upper limits of normal $150 \mathrm{U} / \mathrm{mL}$ ), and a $\gamma$-glutamyl transpeptidase of $48 \mathrm{U} / \mathrm{mL}$ (upper limits of normal $<98 \mathrm{U} / \mathrm{L}$ ). Studies of coagulation revealed an elevated prothrombin time (PT) of $21 \mathrm{~s}$ and an international normalized ratio (INR) of 1.87. Abdominal paracentesis revealed a serum albumin-to-ascites gradient (SAAG) $>1.1 \mathrm{~g} / \mathrm{dL}$, and a total protein concentration of $<2.5 \mathrm{~g} / \mathrm{dL}$, consistent with portal hypertension: The total white blood cell count was $220 / \mathrm{mm}^{3}$, with $22 \%$ neutrophils. These results were compatible with the diagnosis of an uninfected ascites due to portal hypertension as a probable consequence of his cirrhosis. The initial chest radiograph revealed cardiomegaly, bilateral pulmonary edema, and minimal pleural effusions (Fig. 1). Ultrasound scan of the right upper quadrant revealed a nodular liver 


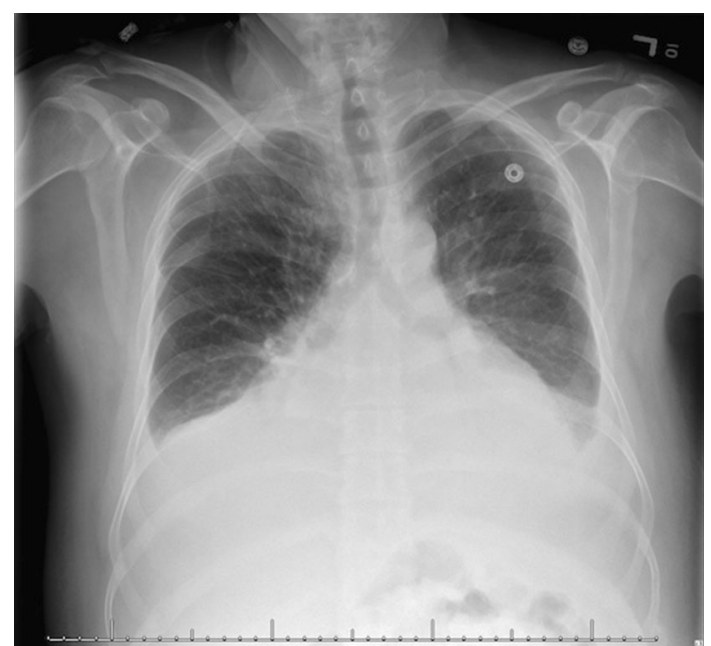

Fig. 1 Chest radiograph with findings of cardiac silhouette enlargement, pleural effusions, and pulmonary edema. Echocardiography confirmed that cardiac silhouette enlargement represented a large pericardial effusion without cardiac chamber enlargement

consistent with cirrhosis with evidence of portal hypertension such as mild splenomegaly and large volume ascites with no space-occupying lesion was identified. Admission pericardiocentesis had an opening pressure of $20 \mathrm{mmHg}$ and, after removal of $1.5 \mathrm{~L}$ of serous pericardial fluid and placement of a drain, a closing pressure of $9 \mathrm{mmHg}$. Pericardial fluid analysis revealed protein concentrations and other characteristics identical to those found in the ascitic fluid: All pericardial cultures were negative, and no organisms were seen. Otherwise, the echocardiogram was relatively normal, as was cardiac function. Over the succeeding $24 \mathrm{~h}, 3 \mathrm{~L}$ of fluid drained from the pericardial sac, as did an additional $3 \mathrm{~L}$ during the subsequent $24-\mathrm{h}$ period. It was suspected that ascitic fluid was exiting through the pericardial drain.

During investigation, a mildly elevated erythrocyte sedimentation rate (ESR) of $28 \mathrm{~mm} / \mathrm{h}$ and serum concentrations of C-reactive protein of $1.1 \mathrm{mg} / \mathrm{dL}$, creatine phosphokinase of $101 \mathrm{U} / \mathrm{L}$, aldolase of $6.1 \mathrm{U} / \mathrm{L}$, and lactate dehydrogenase of $398 \mathrm{U} / \mathrm{L}$ were all noted. Serum lipids and triglycerides were unremarkable. Investigation for the etiology of chronic liver disease revealed a serum ferritin of $113 \mathrm{ng} / \mathrm{mL}$, negative anti-mitochondrial antibody, antismooth muscle antibody, antinuclear antibody, and antiliver kidney microsomal antibodies, and negative virologic evidence for hepatitis A or B. Hepatitis C Ab was positive, but serum hepatitis $C$ virus was undetectable. Serum $\alpha-1$ anti-trypsin and ceruloplasmin, serum copper, and TSH concentrations were normal. Serological investigations for a possible rheumatological disease were unremarkable as well as infectious evaluation for HIV, Lyme disease, and Mycobacterium tuberculosis. A PET/CT scan revealed no FDG-avid focus in any area.
Since decreased pericardial fluid volume was noted on the fourth and fifth hospital days, the pericardial drain was removed. Two days later, he became progressively dyspneic with repeat chest X-ray showing bilateral pleural effusions, cardiomegaly, and diffuses pulmonary edema.

A radionuclide SPECT scan was then performed following injection of $5.11 \mathrm{mCi}{ }^{99 \mathrm{~m}}$ Technetium-labeled, macroaggregated albumin (MAA) into the peritoneal cavity. There was essentially no delay between the infusion of ${ }^{99 \mathrm{~m}}$ Tc-MAA and imaging. Although dynamic imaging was performed, the uptake in the pericardial sac was only faint at $15 \mathrm{~min}$, but clearly visible at $1 \mathrm{~h}$. The most impressive images were obtained at 4-24 h (Fig. 2), where there was circumferential pericardial radiotracer distribution.

\section{Treatment}

Diuretic therapy and continued sodium restriction were initiated in the hope of decreasing formation of ascites and consequently of pericardial effusion, but these measures were unsuccessful. Given ongoing alcohol and illegal drug use, he was not a candidate for liver transplantation. Therefore, a transjugular intrahepatic porto-systemic shunt (TIPS) was performed which greatly relieved his symptoms. A post-procedure chest radiograph was with markedly improved appearance of the cardiac shadow and both lung fields (Fig. 3). Resolution of the ascites and pericardial effusion is illustrated in Fig. 4.

\section{Discussion}

A 41-year-old man with presumed Laennec's cirrhosis and a scar from an upper abdominal stab wound that had required exploratory laparotomy in early 1990s (initially not apparent or mentioned on admission to the Medicine Service) presented with symptoms of pericardial tamponade and ascites. On his most recent admission, placement of a pericardial drain was required through which drained $7.5 \mathrm{~L}$ of fluid. Despite diuretic therapy and sodium restriction during hospitalization, after removal of pericardial drain, he sustained a rapid recurrence of the pericardial effusion. Work-up for numerous etiologies, including infectious, rheumatologic, and neoplastic, were all essentially negative. On later questioning, this patient volunteered that he had failed to recall that his initial stab wound had penetrated his pericardium. A subsequent radionuclide scan revealed a fistulous communication between the peritoneal and pericardial spaces. Failing conventional therapy for ascites and ineligible for liver transplantation, he underwent a potentially lifesaving TIPS procedure with complete resolution of his ascites and his pericardial effusion. 
Fig. 2 Axial and multi-planar reconstructed fused SPECT-CT images show radiotracer uptake within a large pericardial effusion at $4-24 \mathrm{~h}$ postinfusion. No significant ${ }^{99 \mathrm{~m}} \mathrm{Tc}$ MAA activity was present within the pleural effusions, which were felt to be cardiogenic in nature, rather than representing hepatic hydrothorax

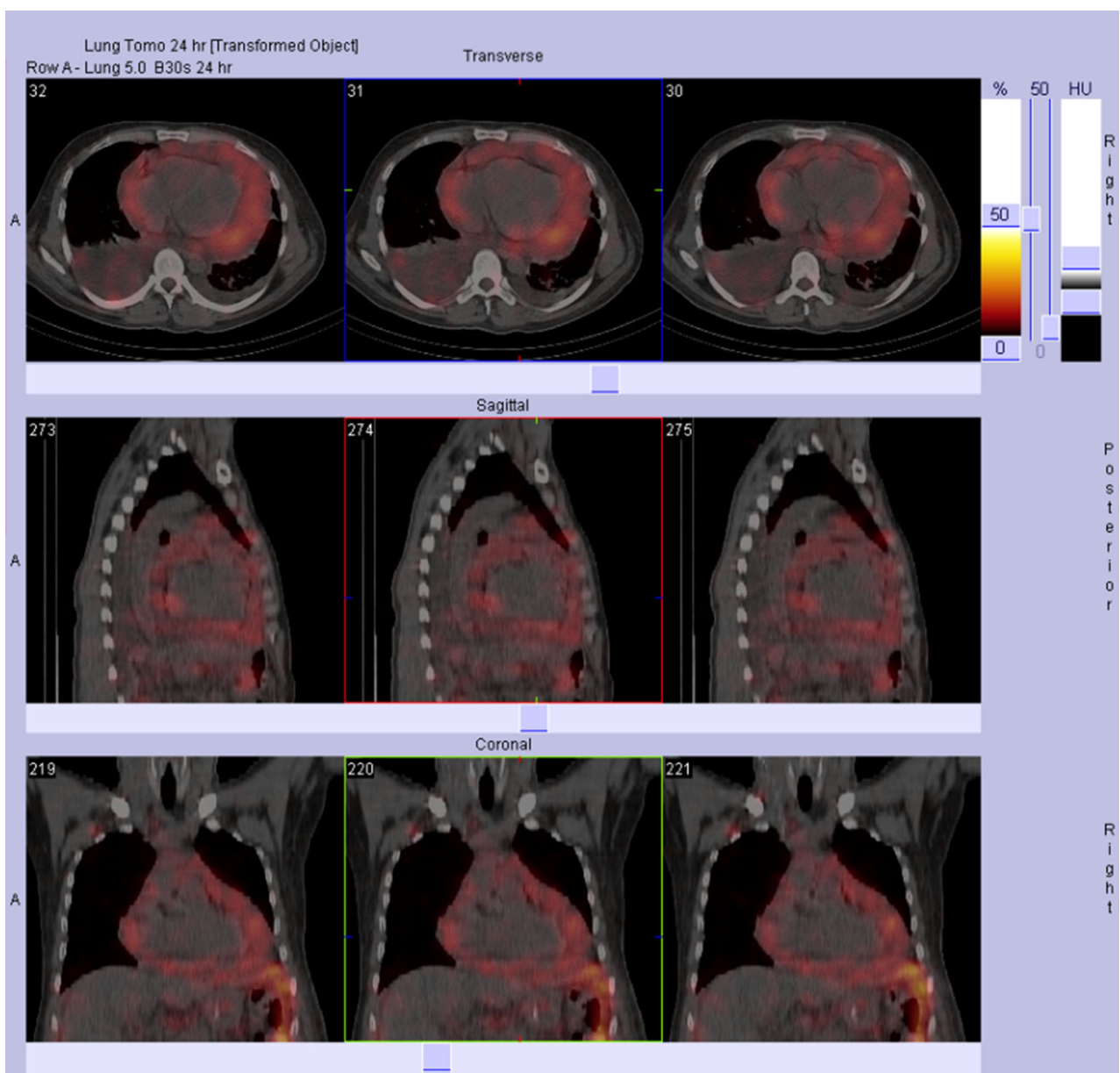

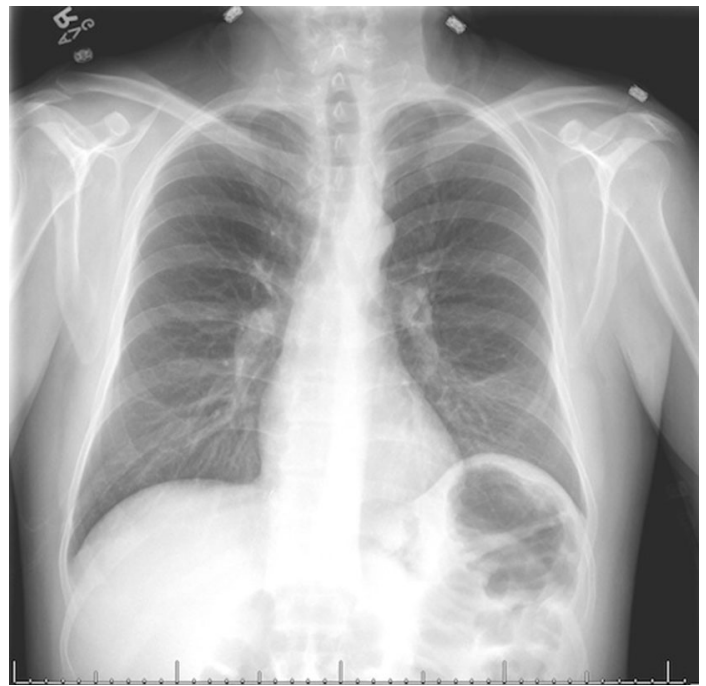

Fig. 3 Post-TIPS chest radiograph shows restoration of a normal cardiac silhouette, resolution of pericardial and pleural effusions, and resolved cardiogenic pulmonary edema

Pericardial effusion resulting from a communication between peritoneum and pericardium is reported very infrequently due to its unusual occurrence. Pleural effusions are more commonly associated with hepatic cirrhosis and ascites as a result of embryological defects in diaphragmatic membrane closure. Peritoneo-pleural communications can occur secondary to congenital fistulae in the lumbocostal triangle of Bodaleck, the right retrosternal triangle of Morgagni or the left sternocostal triangle of Larrey [1]. Under these conditions, ascitic fluid will flow from the peritoneal to the pleural space, given that pressure in the pleural cavity is less than that of the peritoneal cavity, more commonly on the right side, but followed by left. In this setting, pericardial effusion has been reported in $4-10 \%$ of patients with cirrhosis [2] and up to $63 \%$ of patients with decompensated cirrhosis [3] related to the physiological junction of pericardium and pleura. Cheung et al. [4] in discussing a case of hepatic hydropericardium that resolved after orthotopic liver transplantation theorized that there are diaphragmatic and pericardial fenestrations that facilitate accumulation of ascitic fluid in the pericardium. Mild-to-moderate pericardial effusions, with only $16 \mathrm{~mL}$ of fluid, were reported in patients with alcoholic cirrhosis [17 of 27 patients $(63 \%)$ compared with 3 of 28 controls (11\%)] [5].

Badillo et al. [6] reported that all patients with hepatic hydrothorax have advanced liver disease, as defined by a mean model for end-stage liver disease (MELD) score of 

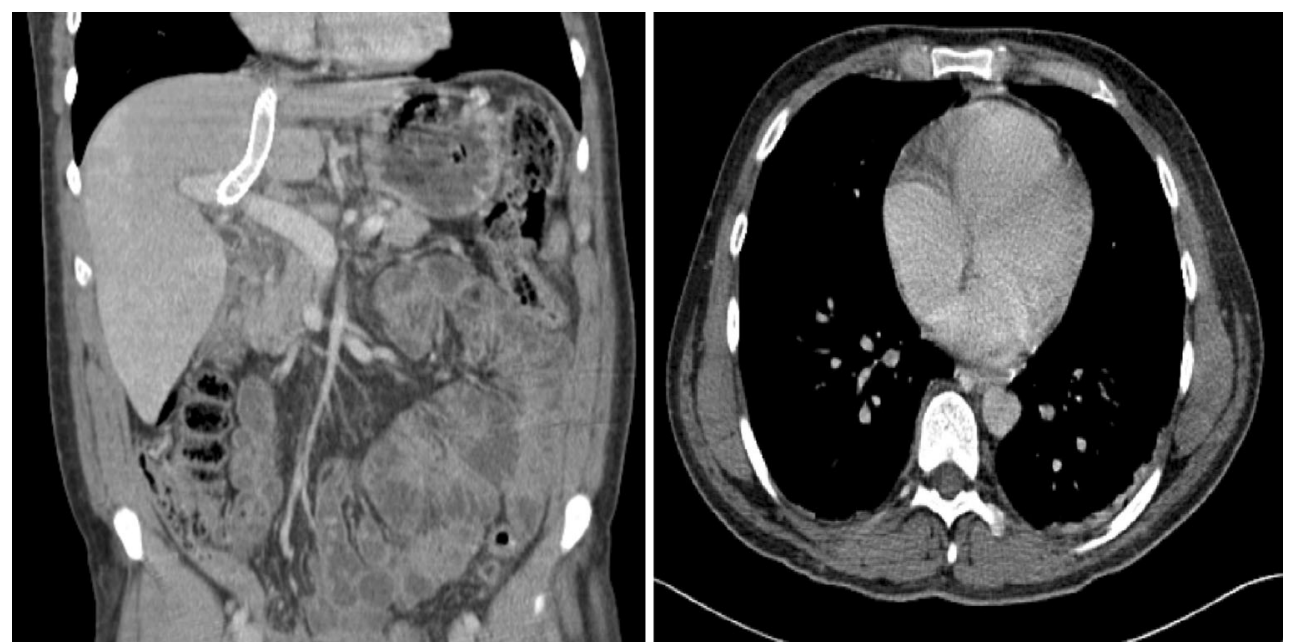

Fig. 4 Axial and multi-planar reformatted coronal CT images, $72 \mathrm{~h}$ after performing TIPS, confirm patency of the porto-systemic shunt and the absence of ascites (left panel), with complete resolution of the pericardial effusion (right panel)

16 at initial evaluation. Most patients $(64 / 77 ; 83 \%)$ reported were managed with diuretics and/or thoracentesis, while $8(10 \%)$ underwent TIPS and $5(7 \%)$ underwent liver transplantation. A total of 44/77 (57\%) patients died during a mean follow-up of 12 months. The average time from initial evaluation to death for all patients, including medical management, TIPS, and liver transplant, was 368 versus 845 days for those who underwent TIPS. Using TIPS in patients with ascites and hepatic hydrothorax has been reported, although infrequently, with prolonged survival [6-9], but little is known about hepatic causes of pericardial effusion.

\section{Summary}

Pericardial effusion causing cardiac tamponade occurred in a previously asymptomatic patient with a history of alcoholic liver disease and upper abdominal trauma, raising the possibility of existence of a peritoneo-pericardial communication. Had the history been given sufficient attention, a plethora of laboratory and imaging studies aimed at excluding multiple etiologies may have been avoided. The definitive test was a radionuclide scan that revealed the communication between the peritoneal and pericardial spaces. It is likely that a fistulous tract between peritoneum and pericardium, from a stab wound in the early 1990s, became manifest only after he developed significant ascites due to decompensated cirrhosis and portal hypertension. The key to relieving the cardiac tamponade, caused by the ascites passing through the fistula, was using TIPS to control the portal hypertension, ascites, and the resulting cardiac tamponade, which proved lifesaving.

Open Access This article is distributed under the terms of the Creative Commons Attribution Noncommercial License which permits any noncommercial use, distribution, and reproduction in any medium, provided the original author(s) and the source are credited.

\section{References}

1. Shumpelick V, Steinau G, et al. Surgical embryology and anatomy of the diaphragm with surgical applications. Surg Clin N Am. 2000;80:213-239.

2. Alberts W, Salem A, et al. Hepatic hydrothorax: cause and management. Arch Intern Med. 1989;142:1462.

3. Naschitz JE, Slobodin G, et al. Heart diseases affecting the liver and liver diseases affecting the heart. Am Heart $J$. 2000;140:111-120.

4. Cheung TK, Tam W, et al. Hepatic hydropericardium. J Gastroenterol Hepatol. 2004;19:109-112.

5. Shah A, Variyam E. Pericardial effusion and left ventricular dysfunction associated with ascites secondary to hepatic cirrhosis. Arch Intern Med. 1988;148:585-588.

6. Badillo R, Rockey DC. Hepatic hydrothorax: clinical features, management, and outcomes in 77 patients and review of the literature. Medicine (Baltimore). 2014;93:135-142.

7. Degawa M, Hamasaki K, et al. Refractory hepatic hydrothorax treated with transjugular intrahepatic portosystemic shunt. $J$ Gastroenterol. 1999;34:128-131.

8. Jeffries MA, Kazanjian S, et al. Transjugular intrahepatic portosystemic shunts and liver transplantation in patients with refractory hepatic hydrothorax. Liver Transpl Surg. 1998;4:416-423.

9. Mitamura K. Further experiments are necessary to determine whether tranjugular intrahepatic portosystemic shunt is the definitive treatment for refractory hepatic hydrothorax. J Gastroenterol. 1999;34:154-156. 